\title{
Deep phenotyping of speech and language skills in individuals with 16p11.2 deletion
}

\author{
Cristina Mei ${ }^{1}$ - Evelina Fedorenko ${ }^{2}$ - David J. Amor ${ }^{1,3,4} \cdot$ Amber Boys $^{1,3} \cdot$ Caitlyn Hoeflin $^{5} \cdot$ Peter Carew ${ }^{1,4}$. \\ Trent Burgess $^{1,3,4} \cdot$ Simon E. Fisher ${ }^{6,7} \cdot$ Angela T. Morgan $\mathbb{B}^{1,4}$
}

Received: 8 May 2017 / Revised: 21 October 2017 / Accepted: 16 January 2018 / Published online: 14 February 2018

(c) European Society of Human Genetics 2018

\begin{abstract}
Recurrent deletions of a $\sim 600-\mathrm{kb}$ region of $16 \mathrm{p} 11.2$ have been associated with a highly penetrant form of childhood apraxia of speech (CAS). Yet prior findings have been based on a small, potentially biased sample using retrospectively collected data. We examine the prevalence of CAS in a larger cohort of individuals with 16p11.2 deletion using a prospectively designed assessment battery. The broader speech and language phenotype associated with carrying this deletion was also examined. 55 participants with $16 \mathrm{p} 11.2$ deletion ( 47 children, 8 adults) underwent deep phenotyping to test for the presence of CAS and other speech and language diagnoses. Standardized tests of oral motor functioning, speech production, language, and non-verbal IQ were conducted. The majority of children (77\%) and half of adults (50\%) met criteria for CAS. Other speech outcomes were observed including articulation or phonological errors (i.e., phonetic and cognitive-linguistic errors, respectively), dysarthria (i.e., neuromuscular speech disorder), minimal verbal output, and even typical speech in some. Receptive and expressive language impairment was present in $73 \%$ and $70 \%$ of children, respectively. Co-occurring neurodevelopmental conditions (e.g., autism) and non-verbal IQ did not correlate with the presence of CAS. Findings indicate that CAS is highly prevalent in children with 16p11.2 deletion with symptoms persisting into adulthood for many. Yet CAS occurs in the context of a broader speech and language profile and other neurobehavioral deficits. Further research will elucidate specific genetic and neural pathways leading to speech and language deficits in individuals with $16 \mathrm{p} 11.2$ deletions, resulting in more targeted speech therapies addressing etiological pathways.
\end{abstract}

Electronic supplementary material The online version of this article (https://doi.org/10.1038/s41431-018-0102-x) contains supplementary material, which is available to authorized users.

Angela T. Morgan

angela.morgan@mcri.edu.au

Murdoch Childrens Research Institute, Melbourne, Australia

2 Department of Psychiatry, Massachusetts General Hospital, Boston, MA, USA

3 Victorian Clinical Genetics Services, Melbourne, Australia

4 The University of Melbourne, Melbourne, Australia

5 Department of Brain and Cognitive Sciences, Massachusetts Institute of Technology, Cambridge, MA, USA

6 Language and Genetics Department, Max Planck Institute for Psycholinguistics, Nijmegen, Netherlands

7 Donders Institute for Brain, Cognition and Behaviour, Nijmegen, Netherlands

\section{Introduction}

A recurrent $\sim 600-\mathrm{kb}$ heterozygous deletion of $16 \mathrm{p} 11.2$, at approximate position $\sim 29.6-30.2 \mathrm{Mb}$ in the reference genome (GRCh37/hg19), is widely acknowledged to affect speech and language development in individuals who carry it [1-4]. Up to $70 \%$ of affected individuals present with some form of communication disorder [5]. Yet there is a paucity of evidence delineating the specific types of speech and/or language disorders associated with the deletion, limiting precise definition of the communication phenotype.

Specificity of phenotyping is important given that childhood apraxia of speech (CAS), a complex diagnosis, has been identified as a core communication deficit in children with the canonical 16p11.2 deletion [2,6-8]. CAS is a severe motor speech disorder affecting the precision and consistency of speech production [9]. The only study to systematically assess speech in children with $16 \mathrm{p} 11.2$ deletion found all nine verbal participants had CAS [2]. These findings are of considerable interest given that the 
prevalence of CAS in the general population is estimated at only $0.01-0.02 \%$ [10]. However, the study was limited by a small and potentially biased sample and the use of retrospectively collected data [2]. Further work is thus required across a larger sample and using prospective assessments carefully designed to conduct differential diagnosis of communication disorder.

At a broader level of communication phenotyping, beyond CAS, even the simple differentiation between speech and language impairments in individuals with 16p11.2 deletion has received little focus [11], with some studies conflating the two. Yet speech production $[12,13]$ is supported by cognitive and neural mechanisms distinct from those that support higher-level language processing [2]. Similarly, there has been a lack of differentiation of expressive versus receptive language abilities, although both are seemingly affected $[6,14,15]$. Moreover, no study has examined performance in specific language subdomains (e.g., semantics, syntax, phonology). Limited evidence is also available for the related outcome of literacy, with reading and writing often examined in case reports $[1,8]$ rather than larger cohorts [5]. Finally, given that language outcomes have often been described in cases selected for the presence of autism spectrum disorder (ASD), deep speech and language phenotyping in a sample unselected for neurodevelopmental conditions is needed to better understand the specificity and prevalence of communication deficits across the more general population of children with $16 \mathrm{p} 11.2$ deletion. The use of specific diagnoses reached by valid methods is essential for clinical management, as these distinct categories necessitate disorder-specific interventions.

Our aims were threefold: (1) Examine the prevalence of CAS in a large unselected cohort of individuals with 16 p11.2 deletion, using a prospectively determined assessment battery; (2) Characterize specific co-occurring speech production and language diagnoses to provide a comprehensive study of the communication phenotype; and (3) Examine the relationship between CAS and other behaviors of language, oral motor function, non-verbal cognition, as well as the presence of neurodevelopmental conditions (e.g., epilepsy and/or seizures, autism) in an attempt to better understand the underlying cognitive architecture associated with $16 \mathrm{p} 11.2$ deletions.

\section{Subjects and methods}

\section{Participants}

Eligibility criteria for probands included a diagnosis of 16 p11.2 recurrent microdeletion $(\sim 600-\mathrm{kb}$ including DOC $2 A$ and TBX6) and an age between $2 ; 11$ and $18 ; 0$ years. Affected siblings and parents of the proband were also eligible. Participants were excluded if they demonstrated an additional copy-number variant, a neurological condition impacting speech, or had taken part in our previously published work [2].

Participants were recruited from two sources: Victorian Clinical Genetics Services (VCGS, Australia) and the 2015 Simons Variation in Individuals Project (VIP) Connect Family Meeting (Washington, US). For the US sample, the study was advertised to families attending the Simons VIP Meeting. Families within Australia were identified via the VCGS database. Probands on the database who met study eligibility (as described above) were contacted. The Human Research Ethics Committees at The Royal Children's Hospital, Melbourne (\#27053), and the Massachusetts Institute of Technology (\#1306005763) approved the study.

\section{Measures}

Participants completed a range of face-to-face clinical measures, as described below. The battery took $\sim 2-2.5 \mathrm{~h}$ to administer. The number of participants contributing to each measure is available as supplemental material. Demographic data (e.g., hearing, vision, gross, and fine motor) were obtained from parents and the Simons VIP.

\section{Aim 1: Prevalence of CAS, and Aim 2: Broader speech and language phenotype speech production (including CAS)}

Differential diagnosis of speech occurred across: articulation disorder (deficit at the phonetic, motoric level of speech, e.g., phonetic distortions such as a lisp or being unable to produce a particular sound); [16] phonological disorder (deficit at the phonemic level reflecting cognitivelinguistic errors e.g., a sound can be produced but is used in incorrect contexts or word positions); [17] dysarthria (impaired neuromuscular execution of speech associated with disruption of tone and/or incoordination of movements, e.g., speech may sound "slurred"); [18] and CAS (impaired planning and programming of speech impacting on precision and consistency, e.g., difficulty initiating and sequencing speech, within speech groping or searching for the correct placement of the tongue and lips) $[9,18]$.

Articulation deficits were evaluated using the GoldmanFristoe Test of Articulation-2 (GFTA-2) [19]. GFTA-2 responses were analyzed for phonological processes and compared to normative data [20] to identify disordered, delayed or age appropriate processes (see supplemental material Table S1 for definitions). This distinction is essential given that disordered phonology (compared to delayed) is associated with persistent speech difficulties and 
Table 1 Features of childhood apraxia of speech (CAS)

\begin{tabular}{ll} 
Children, $n(\%)$ & \\
\hline CAS criteria: & \\
\hline Met & Not met \\
$n=34$ & $n=10$
\end{tabular}

\begin{tabular}{ll} 
Adults, $n(\%)$ & \\
\hline CAS criteria: & \\
\hline Met & Not met \\
$n=4$ & $n=4$
\end{tabular}

\section{Inconsistent errors}

Same C/V different across different words

\begin{tabular}{|c|c|c|c|}
\hline 33 (97) & $1(10)$ & $2(50)$ & $1(25)$ \\
\hline $31(91)$ & $2(20)$ & $3(75)$ & $2(50)$ \\
\hline $11(32)$ & $0(0)$ & - & - \\
\hline $11(32)$ & $0(0)$ & $1(25)$ & $1(25)$ \\
\hline 32 (94) & $4(40)$ & $3(75)$ & $1(25)$ \\
\hline $17(50)$ & $1(10)$ & $0(0)$ & $0(0)$ \\
\hline 32 (94) & $4(40)$ & $3(75)$ & $2(50)$ \\
\hline $31(91)$ & $2(20)$ & $0(0)$ & $0(0)$ \\
\hline $19(56)$ & $0(0)$ & $3(75)$ & $2(50)$ \\
\hline $30(88)$ & $2(20)$ & $2(50)$ & $0(0)$ \\
\hline $6(18)$ & $0(0)$ & $3(75)$ & $0(0)$ \\
\hline $10(29)$ & $0(0)$ & $0(0)$ & $0(0)$ \\
\hline $17(50)$ & $3(30)$ & $0(0)$ & $2(50)$ \\
\hline $23(68)$ & $1(10)$ & $0(0)$ & $4(100)$ \\
\hline $24(71)$ & $1(10)$ & $1(25)$ & $0(0)$ \\
\hline $23(68)$ & $1(10)$ & $3(75)$ & $1(25)$ \\
\hline $21(62)$ & $0(0)$ & $0(0)$ & $0(0)$ \\
\hline $23(68)$ & $4(40)$ & $3(75)$ & $2(50)$ \\
\hline $17(50)^{\mathrm{b}}$ & $2(20)^{\mathrm{c}}$ & $3(75)$ & $1(25)$ \\
\hline 33 (97) & $2(20)$ & $2(50)$ & $0(0)$ \\
\hline $30(88)$ & $5(50)$ & $2(50)$ & $1(25)$ \\
\hline $22(65)$ & $1(10)$ & $3(75)$ & $1(25)$ \\
\hline $30(88)$ & $4(40)$ & $1(25)$ & $2(50)$ \\
\hline \multirow[t]{2}{*}{34 (100) } & $0(0)$ & $4(100)$ & $0(0)$ \\
\hline & $6(60)$ & & $4(100)$ \\
\hline $0(0)$ & $1(10)$ & $0(0)$ & $0(0)$ \\
\hline $0(0) 0(0)$ & $2(20)$ & $0(0) 0(0)$ & $0(0)$ \\
\hline
\end{tabular}

Same word/syllable different on repetitions

Inconsistency $>40 \%$ on the DEAP ${ }^{\mathrm{a}}$

Lengthened \& disrupted coarticulatory transitions

Speech motor behaviors, including groping during

sound production

Difficulty sequencing phonemes \& syllables

Voicing errors

Errors increase with word length \& phonological

complexity

Syllable segregation

Difficulty achieving initial articulatory configurations

or transitory movement gestures

Difficulty maintaining syllable integrity

Repetitions of sounds \& syllables

Epenthesis/intrusive schwa

Metathesis

Addition errors

Frequent omissions $(>10)$

Prolongation errors

Nonphonemic productions/distorted substitutions

Hypernasality/nasal emissions

Slowed \& disrupted DDK sequence

Inappropriate prosody

Equal stress or lexical stress errors

Altered suprasegmentals

Prolongation errors

Slow rate

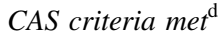

2

1

0

C/V consonant/vowel, DDK diadochokinesis.

${ }^{\mathrm{a}}$ Not completed by 12 children and all of the adults. ${ }^{\mathrm{b}}$ Not completed by 12/34. ${ }^{\mathrm{c}}$ Not completed by $2 / 34$.

${ }^{\mathrm{d}}$ Unknown for one child (who did not meet the criteria for CAS) since their data was obtained from clinical reports.

impaired phonological awareness and literacy [21, 22]. Dysarthria was diagnosed based on (1) the presence of oromotor dysfunction (i.e., impaired Focal Oromotor Control based on speech and non-speech tasks, as described below) signaling impaired neuromuscular tone or coordination of movements; and (2) concomitant deviant perceptual speech features rated during a 10-min conversational speech sample, affecting articulation, resonance, prosody, respiration, and/or phonation.

For CAS, diagnostic criteria were adapted from Murray et al. [23, 24] based on three American Speech and Hearing Association consensus-based criteria [9], as in our previous work [2]: (1) inconsistent production of the same word; (2) lengthened and disrupted coarticulatory transitions; and 3) 
prosodic errors (see Table 1). The same criteria were used for child and adult participants given the absence of diagnostic criteria for adults with CAS. Inconsistent production was examined using the standardized Inconsistency subtest of the Diagnostic Evaluation of Articulation and Phonology (DEAP) [20]. Prosodic, syllable and sound sequencing errors typifying disrupted co-articulatory transitions were analyzed using the Single-Word Test of Polysyllables [24, 25]. Overall speech accuracy ratings of Percent Consonants Correct (PCC) and Percent Vowels Correct (PVC) were also derived from this tool. Where this test could not be completed $(n=8)$, speech accuracy ratings were calculated using the GFTA-2. PCC scores were categorized by severity: mild (>85), mild-moderate (65-85), moderatesevere (50-64), and severe (<50) [26]. The 10-minute conversational speech sample was also analyzed to identify further features of CAS (see Table 1) [2, 24, 25].

\section{Oral motor function}

Oral motor function was assessed in children aged $\geq 3$ years using the Verbal Motor Production Assessment for Children (VMPAC) [27], the most psychometrically robust oral motor assessment available for children [28]. Three scales were administered: Global Motor Control, a measure of neuromotor innervation to peripheral muscles in the torso, neck, head and face; Focal Oromotor Control assessing control of the jaw, lips, face and tongue during speech and non-speech tasks; and Sequencing, examining performance of oral and speech movements in sequential order.

Children $<3$ years were evaluated using the Oral and Speech Motor Control Protocol (OSMCP) [29]. The DEAP Oromotor subtest [20], which includes tasks examining isolated and sequenced non-speech movements, was used for two children unable to complete the VMPAC due to reduced attention. Oromotor abilities of adults ( $>18$ years) were measured using the Frenchay Dysarthria Assessment-2 (FDA-2) [30]. These tools assess both speech and nonspeech oral motor tasks.

\section{Language \& literacy}

Australian participants $<21$ years were evaluated with the Clinical Evaluation of Language Fundamentals (CELFPreschool 2 or CELF-4, depending on age) [31, 32]. The CELF Core Language Score, reflecting overall receptive and expressive language competence, was used to identify impaired language. Children $\leq 3$ years were assessed using the Preschool Language Scale-5 (PLS-5) [33], a measure of receptive and expressive language. Due to time constraints, language functioning of the US child cohort $(n=5)$ was measured using the CELF-5 Recalling Sentences subtest [34]. Deficits in receptive and expressive language were recorded based on the CELF Core Language subtests, with impairments in specific subdomains (i.e., semantics, syntax, morphology) further identified. For those who completed the PLS-5, deficits were based on participants' overall scores (Auditory Comprehension and Expressive Communication) and the Profile form. Auditory short-term memory was also examined in the Australian cohort aged $>5$ years ( $n=26$ ) using the Forwards Number Repetition subtest of the CELF-4.

Seven of 8 adults were examined using the Peabody Picture Vocabulary Test-4 (PPVT-4) [35], a measure of receptive vocabulary, and the Test for Reception of Grammar-2 (TROG-2) [36], a measure of grammatical comprehension. The Nonword Repetition subtest of the Comprehensive Test of Phonological Processing-2 (CTOPP-2) [37] was completed by children $(n=29)$ and adults $(n=7)$ as an indicator of phonological processing and awareness, early precursors of literacy. Literacy outcomes of the Australian cohort $(n=26)$ were measured using the Word Reading and Spelling subtests of the Wide Range Achievement Test-4 (WRAT-4) [38].

\section{Aim 3: Associations between CAS and other neurologic cognitive-behavioral domains}

Information regarding additional diagnoses (e.g., epilepsy, autism, attention deficit hyperactivity disorder (ADHD), gross and fine motor impairment) was obtained from parents and the Simons VIP. Non-verbal cognition was measured with the Wechsler Abbreviated Scale of Intelligence-2 (WASI-2) [39] for participants $>6$ years $(n=38)$ or Kaufman Brief Intelligence Test-2 (K-BIT-2) [40] for those $<6$ years $(n=2)$.

\section{Analysis}

Results for the Australian and US cohorts were combined given that demographic characteristics were comparable (Table 2). Where different measures were used across children, scores from measures examining similar constructs were grouped where possible (e.g., CELF-4, PLS-5). For Aim 1, CAS ratings were completed by CM. A second speech pathologist (AM), who was blinded to CAS status, also completed ratings for $n=15, \sim 30 \%$ of the sample, to confirm CAS diagnoses. For Aim 2, data were transcribed and analyzed, and standard scores were computed for all relevant tests by $\mathrm{CM}$ to provide a differential diagnosis of speech, language, and literacy.

For Aim 3, the relationship between CAS and children's broader phenotype (e.g., autism, ADHD, epilepsy and/or seizures, non-speech oromotor) was examined using the Fisher's exact test. The Mann-Whitney U t-test was used to compare the language, auditory short-term memory and 
Table 2 Participant characteristics

\begin{tabular}{|c|c|c|c|}
\hline & \multicolumn{2}{|l|}{ Children } & \multirow[b]{2}{*}{ Adults $(n=8)$} \\
\hline & $\begin{array}{l}\text { Australian }(n=40) \\
n(\%)\end{array}$ & US $(n=7)$ & \\
\hline Age, mean (SD) & $8.27(1.85)$ & $9.84(4.41)$ & $39.85(9.10)$ \\
\hline Female & $14(35)$ & $4(57)$ & $6(75)$ \\
\hline \multicolumn{4}{|l|}{ Inheritance } \\
\hline de novo & $15(38)$ & $3(43)$ & $1(13)$ \\
\hline Inherited & $10(25)$ & $1(14)$ & $0(0)$ \\
\hline Unknown & $15(38)$ & $3(43)$ & $7(88)$ \\
\hline \multicolumn{4}{|c|}{ Intellectual disability } \\
\hline Present & $17(43)$ & $1(14)$ & $1(13)$ \\
\hline Absent & $20(50)$ & $4(57)$ & $7(88)$ \\
\hline Unknown & $3(8)$ & $2(29)$ & $0(0)$ \\
\hline \multicolumn{4}{|c|}{ Epilepsy and/or seizures } \\
\hline Present & $16(40)$ & $4(57)$ & $2(25)$ \\
\hline Absent & $24(60)$ & $2(29)$ & $6(75)$ \\
\hline Unknown & $0(0)$ & $1(14)$ & $0(0)$ \\
\hline \multicolumn{4}{|l|}{ Autism } \\
\hline Present & $17(43)$ & $1(14)$ & $0(0)$ \\
\hline Absent & $23(58)$ & $5(71)$ & $8(100)$ \\
\hline Unknown & $0(0)$ & $1(14)$ & $0(0)$ \\
\hline \multicolumn{4}{|l|}{$A D H D^{\wedge}$} \\
\hline Present & $6(15)$ & $3(43)$ & $0(0)$ \\
\hline Absent & $33(83)$ & $2(29)$ & $8(100)$ \\
\hline Unknown & $1(3)$ & $2(29)$ & $0(0)$ \\
\hline \multicolumn{4}{|c|}{ Hearing impairment } \\
\hline Present & $8(20)$ & $1(14)$ & $2(25)$ \\
\hline Absent & $32(80)$ & $4(57)$ & $6(75)$ \\
\hline Unknown & $0(0)$ & $2(29)$ & $0(0)$ \\
\hline \multicolumn{4}{|l|}{ Vision impairment } \\
\hline Present & $12(30)$ & $1(14)$ & $4(50)$ \\
\hline Absent & $27(68)$ & $2(29)$ & $4(50)$ \\
\hline Unknown & $1(3)$ & $4(57)$ & $0(0)$ \\
\hline \multicolumn{4}{|l|}{ Hypotonia } \\
\hline Present & $16(40)$ & $3(43)$ & $1(13)$ \\
\hline Absent & $21(53)$ & $3(43)$ & $3(38)$ \\
\hline Unknown & $3(8)$ & $2(29)$ & $4(50)$ \\
\hline \multicolumn{4}{|c|}{ Gross motor impairment } \\
\hline Present & $26(65)$ & $3(43)$ & $4(50)$ \\
\hline Absent & $14(35)$ & $1(14)$ & $4(50)$ \\
\hline Unknown & $0(0)$ & $3(43)$ & $0(0)$ \\
\hline \multicolumn{4}{|c|}{ Fine motor impairment $t^{\wedge}$} \\
\hline Present & $30(75)$ & $1(14)$ & $2(25)$ \\
\hline Absent & $10(25)$ & $4(57)$ & $5(63)$ \\
\hline Unknown & $0(0)$ & $2(29)$ & $1(13)$ \\
\hline \multicolumn{4}{|l|}{ Oral cleft } \\
\hline Present & $1(3)^{\mathrm{a}}$ & $0(0)$ & $0(0)$ \\
\hline Absent & $39(98)$ & $3(43)$ & $8(100)$ \\
\hline
\end{tabular}

Table 2 (continued)

\begin{tabular}{llll}
\hline & \multicolumn{2}{l}{ Children } & \\
\cline { 2 - 3 } & $\begin{array}{l}\text { Australian }(n=40) \\
n(\%)\end{array}$ & US $(n=7)$ & Adults $(n=8)$ \\
\hline Unknown & $0(0)$ & $4(57)$ & $0(0)$ \\
$\begin{array}{l}\text { Tongue tie } \\
\text { Present }\end{array}$ & $4(10)$ & $0(0)$ & $1(13)$ \\
No & $33(83)$ & $0(0)$ & $6(75)$ \\
Unknown & $3(8)$ & $7(100)$ & $1(13)$ \\
\hline
\end{tabular}

${ }^{a}$ Submucous cleft palate.

$\wedge$ Differences between child cases (Australian and US cohorts) significant at 0.05 level (Fisher's exact test).

non-verbal IQ scores of children with and without CAS. The association between CAS and speech-related oromotor impairment was examined using the Spearman's rank correlation. This test was also used to investigate the correlation between language and non-verbal IQ.

\section{Results}

Altogether, 55 participants were recruited: 47 Australian (40 children, 7 adults) and 8 American (7 children, 1 adult). Sample characteristics are detailed in Table 2. Age of participants ranged from $2 ; 11$ to $17 ; 9$ years for probands $(n=44)$ and child siblings $(n=3)$ and $20 ; 4$ to $48 ; 2$ years for the remaining affected adult siblings $(n=1)$ and parents $(n=7)$. Of the child sample, $51 \%(24 / 47)$ were currently receiving speech and/or language therapy while 38\% (18/ 47) had received this type of therapy in the past.

As not all participants completed each assessment (due to factors such as age, time constraints, compliance), the denominators presented below vary. Denominators used reflect the number of participants who completed each assessment, as detailed in the supplemental material (Table S2).

\section{Aim 1: Prevalence of CAS, and Aim 2: Broader speech and language phenotype speech production}

Three child participants were excluded from speech analyses due to minimal verbal output $(n=2)$ or missing data $(n=1)$. Of remaining participants, 89\% (39/44) had impaired speech (Fig. 1). Children demonstrated a range of PCC severity ratings: mild $(51 \%, 22 / 43)$, mild-moderate $(33 \%, 14 / 43)$, moderate-severe $(9 \%, 4 / 43)$, and severe $(2 \%$, 1/43). PCC data were not available for one child.

Diagnostic criteria for CAS were met by $77 \%$ (34/44) of children (Table 1). Only 29\% (10/34) of this sample had 
Fig. 1 Prevalence of speech production disorders

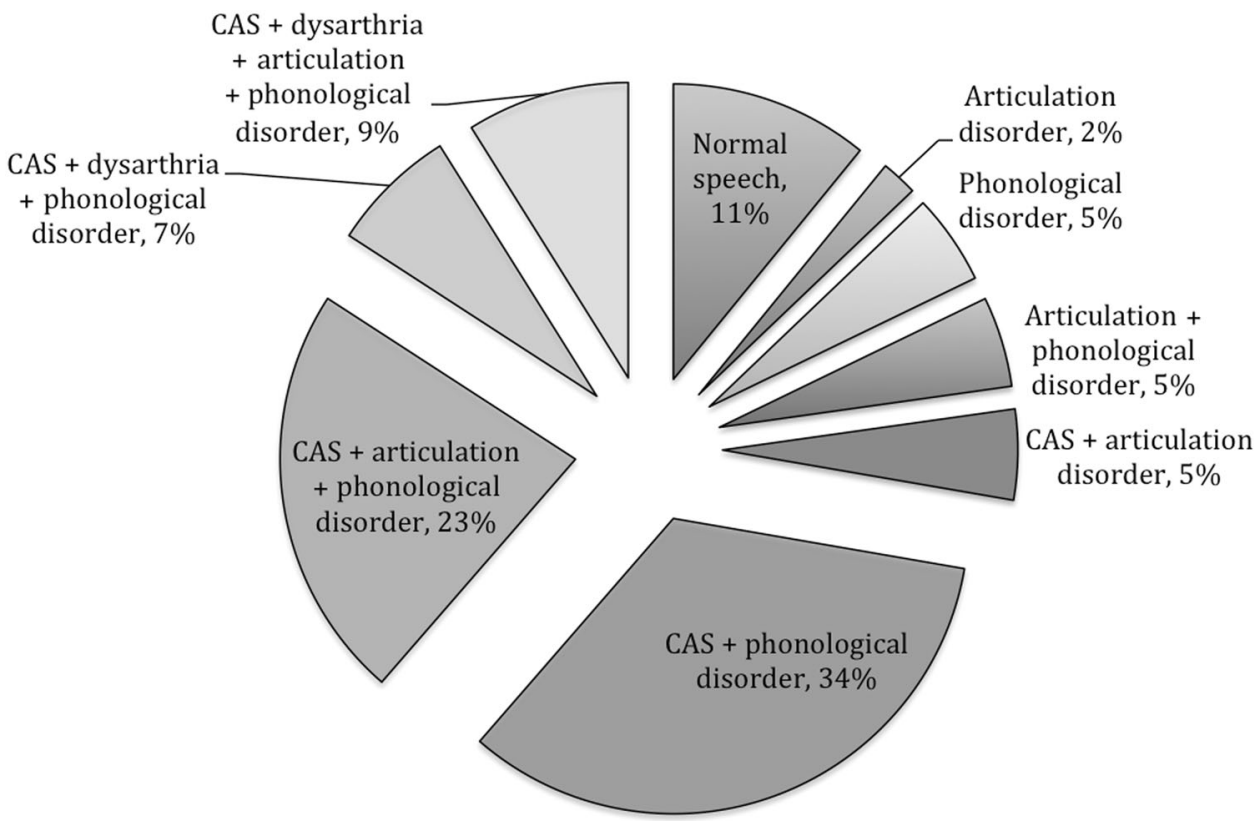

previously received a clinical diagnosis of CAS. Whilst CAS was prevalent, articulation and phonological speech errors were also common, present in 43\% (19/44) and 82\% (36/44) of children, respectively. Of the 19 children with articulation errors (e.g., interdental or lateral fricatives, labiodental production of bilabials), 11 had structural deficits likely to be causing the phonetic distortion (e.g., malocclusion, submucous cleft palate, misaligned teeth). Phonological processes were classified as delayed in $36 \%$ (16/44) and disordered in $45 \%$ of children (20/44) (supplemental material Table S3).

For children, CAS occurred in conjunction with both articulation and phonological errors $(23 \%, 10 / 44)$, phonological errors alone $(34 \%, 15 / 44)$, articulation errors alone $(5 \%, 2 / 44)$ or dysarthria with co-occurring articulation and/ or phonological errors $(16 \%, 7 / 44)$. Five of the ten children without a CAS diagnosis had other forms of speech impairment; isolated phonological $(n=2)$ or articulation errors $(n=1)$, and co-occurrence of these errors $(n=2)$.

For the adult cases, 50\% (4/8) met the CAS diagnostic criteria (Table 1). Three adults presented with articulation errors (i.e., interdental fricatives) and/or phonological processes involving 'th' (i.e., stopping, fronting), respectively used by $25 \%(2 / 8)$ of adults (one demonstrated co-occurring articulation and phonological errors). The remaining five cases showed no errors during less demanding speech tasks (e.g., conversation, GFTA-2), but produced one or more errors (e.g., metathesis, epenthesis, assimilation) during more complex tasks such as producing polysyllables. PCC speech severity ratings were mild in all impaired adult cases $(71 \%, 5 / 7)$.

\section{Oromotor}

In the Australian child cohort, oromotor functioning was impaired in $85 \%$ (28/33). Oromotor functioning was not assessed in 14 children. Thirty children completed the Global Motor Control scale of the VMPAC, with performance classified as within normal limits for $27 \%(8 / 30)$, moderately impaired for $23 \%$ (7/30), and severely impaired for $50 \%$ (15/30). Within this scale, impairments were often characterized by reductions in tongue strength $(53 \%, 16 /$ $30)$, soft palate contraction $(47 \%, 14 / 30)$ and smoothness or range of movement $(33 \%, 10 / 30)$.

The Focal Oromotor Control scale, was completed by 29 children with impaired functioning seen in $76 \%(22 / 29)$. Severity of impairment was classified as mild (7\%, 2/29), moderate $(10 \%, 3 / 29)$, or severe $(59 \%, 17 / 29)$. Poor performance during single non-speech oromotor movements reflected impaired lingual movements $(48 \%, 14 / 29$; e.g., reduced tongue elevation and lateralization), labial-facial movements $(31 \%, 9 / 29$; e.g., poor lip rounding and, in two cases, asymmetry), and mandibular control (10\%, 3/29; e.g., reduced jaw excursion and stability). Impaired double nonspeech oromotor movements (e.g., "smile and kiss") were common $(45 \%, 13 / 29)$, typified by impaired transition and precision of movements. With regard to speech-related oromotor movements, deficits were frequently noted during the production of triple oromotor sequences (e.g., /a-m-u/; $72 \%, 21 / 29)$ and words/sentences (e.g., "bow, toe, go"; $74 \%, 20 / 27)$. In comparison, impaired single (e.g., /a/) and double speech movements (e.g., /a-u/) were seen in $55 \%$ (16/29) and 45\% (13/29), respectively. 
Table 3 Language, literacy and cognitive outcomes

\begin{tabular}{|c|c|c|c|c|}
\hline & \multicolumn{2}{|c|}{ Child cases $(n=47)$} & \multicolumn{2}{|c|}{ Adult cases $(n=8)$} \\
\hline & Impaired n (\%) & $\begin{array}{l}\text { Mean, SD } \\
\text { (Range) }\end{array}$ & Impaired n (\%) & $\begin{array}{l}\text { Mean, SD } \\
\text { (Range) }\end{array}$ \\
\hline \multicolumn{5}{|l|}{ Language } \\
\hline Core language $\mathrm{e}^{\mathrm{a}, \mathrm{d}}$ & $26 / 31(84)^{b}$ & $\begin{array}{l}68.9,16.7 \\
(40-112)\end{array}$ & 1/1 (100) & 79 \\
\hline Receptive semantics & $22 / 27(81)$ & - & $0 / 1(0)$ & - \\
\hline Receptive syntax & $3 / 3(100)$ & - & - & - \\
\hline Expressive semantics & $18 / 22(82)$ & - & $1 / 1(100)$ & - \\
\hline Expressive morphology & 11/11 (100) & - & - & - \\
\hline $\begin{array}{l}\text { Expressive semantics and } \\
\text { morphosyntax }\end{array}$ & $25 / 30(83)$ & - & $0 / 1(0)$ & - \\
\hline PPVT-4 ${ }^{\mathrm{a}, \mathrm{e}}$ & $1 / 1(100)$ & 20 & $3 / 6(50)$ & $82.7,5.5$ (74-91) \\
\hline TROG- $^{\mathrm{a}}$ & & - & $2 / 5(40)$ & $80.6,9.1(67-90)$ \\
\hline \multicolumn{5}{|l|}{ Literacy $(W R A T-4)^{\mathrm{a}}$} \\
\hline Word Reading & $11 / 20(55)$ & $\begin{array}{l}82.8,17.9 \\
(59-128)\end{array}$ & $2 / 6(33)$ & $\begin{array}{l}79.0,15.8 \\
(56-96)\end{array}$ \\
\hline Spelling & $8 / 18(44)$ & $\begin{array}{l}81.8,13.8 \\
(55-106)\end{array}$ & $2 / 6(33)$ & $\begin{array}{l}82.7,15.7 \\
(61-107)\end{array}$ \\
\hline Non-verbal $I Q^{\mathbf{a}, \mathbf{c}}$ & & $\begin{array}{l}79.5,14.2 \\
(61-112)\end{array}$ & - & $76.4,6.3(69-83)$ \\
\hline High average & $1 / 33(3)$ & - & $0 / 7(0)$ & - \\
\hline Average & $7 / 33(21)$ & - & 0/7 (0) & - \\
\hline Low average & $6 / 33(18)$ & - & $3 / 7(43)$ & - \\
\hline Borderline & $8 / 33(24)$ & - & 2/7 (29) & - \\
\hline Extremely low & $11 / 33(33)$ & - & 2/7 (29) & - \\
\hline Auditory short-term memory ${ }^{\mathbf{f}, \mathbf{h}}$ & $20 / 25(80)$ & $5.2,1.9(1-9)$ & - & - \\
\hline CTOPP $-2^{\mathbf{h}}$ & $32 / 34(94)$ & $4.4,2.4(1-9)$ & $7 / 7(100)$ & $2.7,2.0(1-6)$ \\
\hline
\end{tabular}

CTOPP Comprehensive Test of Phonological Processing, PPVT Peabody Picture Vocabulary Test, TROG Test for Reception of Grammar, WRAT Wide Range Achievement Test.

${ }^{a}$ Normative mean $=100\left(\right.$ SD 15). ${ }^{\mathrm{h}}$ Normative mean $=10($ SD 3). Scores greater than 1 SD below the normative mean were classified as impaired except for non-verbal IQ, which was classified as: high average (110-119), average (90-109), low average (80-89), borderline (70-79) and extremely low $(<70)$

${ }^{\mathrm{b}}$ US cohort not included as they only completed the Recalling Sentences subtest. Language was unable to be assessed in 7 of the Australian children due to reasons such as the cooperation of the child, profound language difficulties, or the absence of a recent language assessment for children whose data were obtained from clinical reports.

${ }^{c}$ Cognitive testing not possible for 14 children (due to the child's age or capabilities) and 1 adult.

${ }^{\mathrm{d}}$ Measured using the CELF-4, CELF-P2 or PLS-5.

e 1 child completed the PPVT-4, a measure of receptive vocabulary, as they were unable to complete the CELF-4 due to severe speech and language impairment.

${ }^{\mathrm{f}}$ Measured using the CELF-4 Number Repetition-Forwards subtest (Australian cohort only).

${ }^{g}$ Not completed by 5 children who were unable to complete the sample items.

Twenty-nine children completed the Sequencing scale. Performance on this scale revealed the greatest difficulty, with severe impairment noted in $79 \%$ (23/29) of children. Mild and moderate impairments were infrequent $(14 \%$, $4 / 29$, and $7 \%, 2 / 29)$ as were intact sequencing skills (14\%, 4/29). Sequencing deficits were seen across all tasks (i.e., sequencing of non-speech oromotor movements, phonemes, and words).
Oromotor functioning was assessed in 6 of the 8 adults, with all showing some degree of impaired laryngeal and tongue control (during speech and/or non-speech tasks). Common features included reductions in phonation time, volume control, and non-speech and speech tongue movements (elevation and alternating sequences). Palatal and lip deficits were also common, both occurring in $67 \%(4 / 6)$ of adults. These deficits reflected poor palatal movement 
during phonation and reduced alternating lip movements during speech. Reflexes (e.g., cough, swallow) and intelligibility were relatively spared (both areas impaired in 1/6 cases). Reduced respiratory support for speech was observed in two adults although this was associated with a chest infection.

\section{Language, cognition \& literacy}

For the child cases, language was not evaluated in seven due to poor child compliance $(n=4)$, severity of verbal impairment $(n=1)$, or missing data $(n=2)$. Of those assessed, 83\% (33/40) had a language impairment, with Core Language Scores (i.e., overall receptive-expressive abilities) greater than two standard deviations below the normative mean (Table 3). Language impairment in children commonly affected both expressive and receptive abilities $(60 \%, 24 / 40)$ rather than only expressive $(5 \%, 2 /$ $40)$ or only receptive $(3 \%, 1 / 40)$ abilities. Differences in expressive and receptive language were not examined in 6 children as only one or the other domain was evaluated due to severe expressive language difficulties $(n=2)$, selective mutism $(n=1)$, or time constraints $(n=3)$. Impairments were present across the subdomains of semantics, syntax, and morphology (Table 3). Non-verbal IQ and literacy scores for children were greater than one standard deviation below the normative mean (Table 3). Auditory short-term memory (measured using the CELF-4) and phonological memory (based on the CTOPP-2) were respectively impaired in $80 \%(20 / 25)$ and $94 \%(32 / 34)$ of children (Table 3).

For the adults, $86 \%(6 / 7)$ had impaired language, with performance on the PPVT-4 and TROG-2 greater than one standard deviation below the mean (Table 3). Language was not assessed with one adult. Non-verbal IQ and literacy scores for this group were two standard deviations below the normative mean (Table 3 ).

\section{Aim 3: Associations between CAS and other neurologic cognitive-behavioral domains}

Results from the Fisher's exact test did not identify an association between CAS status in the children and autism $(p=0.15)$, ADHD $(p=>0.999)$, gross or fine motor function ( $p=0.27$, and $p=0.70$, respectively), epilepsy and/or seizures $(p=0.47)$, Similarly, the Mann-Whitney U t-test did not reveal an association between CAS and language $(\mathrm{U}=64, p=0.12)$ and non-verbal IQ $(\mathrm{U}=69, p=$ 0.15 ), although CAS was associated with poor short-term auditory memory $(\mathrm{U}=21, p=0.01)$.

The association between CAS and non-speech oromotor function in children, measured by the Fisher's exact test, was not statistically significant $(p=0.55$ for mandibular control, $p=0.37$ for labial-facial control, and $p=0.39$ for tongue function). Similarly, results from the Spearman's rank test revealed that Focal Oromotor Control percentage scores (i.e., speech and non-speech oromotor performance) were not correlated with GFTA-2 standard scores for children with CAS $(r=0.13, p=0.58)$. This pattern suggests that articulation was not associated with oromotor performance for this group. Performance on the Sequencing scale was related to DEAP Inconsistency scores $(r=-0.93, p=$ $<0.001)$ but not GFTA-2 scores $(r=0.26, p=0.26)$ for children with CAS, indicating that children with poorer sequencing abilities (during speech and non-speech tasks) produced a higher number of inconsistent speech errors.

Children's language scores (reflecting overall receptive and expressive language competence) were not correlated with non-verbal IQ $(r=-0.08, p=0.73)$, even when children with hearing impairment were excluded $(r=$ $-0.04, p=0.86$ ). Notably, the language abilities of 6 children were $\geq 2$ standard deviations below their non-verbal IQ.

\section{Discussion}

In line with our previous work [2], we found that CAS is prevalent $(77 \%)$ in individuals with canonical 16p11.2 deletions. Further, our findings suggest that speech and/or language disorder is more common than previously estimated in this population, with $98 \%$ of children demonstrating some form of speech or language impairment and a significant proportion (57\%) having co-occurrence of these deficits. Other neurobehavioural conditions (e.g., epilepsy and/or seizures, autism) were not correlated with a diagnosis of CAS.

Although most children (89\%) demonstrated impaired speech, severity ratings were mild or mild-moderate for the majority of the cohort (i.e., PCC ratings $>65$ ), and only two cases were minimally verbal. Interestingly, only $29 \%$ of children with CAS had previously received a clinical diagnosis of CAS (despite all previously having seen a SLP), suggesting potential misdiagnosis in the clinical community and, consequently, lack of targeted intervention of motor planning and programming deficits, as needed for improving these children's outcomes. This result is not surprising considering the variable criteria used by clinicians for the diagnosis of CAS [41]. Our findings highlight the need for clinicians to systematically test for the presence of CAS based on the ASHA consensus criteria in children with $16 \mathrm{p} 11.2$ deletion to improve management of speech in this population. Further, our data show that CAS commonly occurs with other speech disorders (dysarthria, articulation, and phonological), hence a careful differential diagnosis is required and clinical problem solving needed to 
therapeutically target the specific level(s) of deficit. In addition to managing surface behaviors, health professionals may consider referring children with CAS for clinical genetic testing if indicated to determine potential underlying causes such as $16 \mathrm{p} 11.2$ deletion.

In relation to language, a fairly consistent profile was demonstrated in that deficits involved both receptive and expressive language. This is in line with previous reports where a mixed receptive-expressive language impairment profile has been associated with $16 \mathrm{p} 11.2$ deletion $[6,14$, 15]. Here, however, we highlight that the language deficit is not restricted to a particular subdomain, rather it is generalized affecting morphology, syntax and semantics. This finding is shared by other syndromes associated with intellectual disability (e.g., Floating Harbour and Kabuki) $[42,43]$. Our findings further refine the phenotype associated with $16 \mathrm{p} 11.2$ deletion by revealing that it is not linked to a syndrome-specific language profile where only specific subdomains are affected.

Although it is probable that children's broader cognitive profile is related to their language attainment given their shared genetic bases [44] mean language scores were nearly one standard deviation below non-verbal IQ. In contrast, children's literacy outcomes, as measured by the WRAT-4, were a relative strength and more commensurate with average non-verbal IQ performance. This differs from previous research where strengths in literacy were not as apparent in comparison to language [5]. For instance, in Hanson et al. [5] word reading abilities were, on average, just below verbal IQ (76 and 79, respectively) whereas here, a greater split between word reading and language abilities was apparent (83 and 69, respectively). This difference could potentially be due to differences in measures or sample characteristics (e.g., in Hanson et al. [5] $46 \%$ had language impairment whereas here it occurred in $83 \%$ of children).

With regard to adults, all had mild speech severity ratings and typically only issues during more demanding speech motor tasks (e.g., polysyllabic words). Notably, the diagnostic criteria for CAS were met by $50 \%$ of adults with the remaining cases showing some symptoms despite not meeting the pre-defined criteria for a formal diagnosis. Importantly, the phenotype of CAS differed between adults and children, consistent with the broader CAS literature [45]. Specifically, children demonstrated features such as syllable segregation and voicing errors that were not seen in adults. Although CAS is recognized as a persistent speech disorder, how it evolves during adulthood remains poorly understood, including for individuals with 16p11.2 deletions. The cross-sectional design of the current study makes it difficult to infer the progression of speech disorders for this population, necessitating the need for future large scale prospectively designed longitudinal studies.
In terms of associated factors, CAS occurred independent of other neurobehavioural conditions, such as autism, ADHD, and epilepsy and/or seizures in children. Nonverbal IQ was also not predictive of CAS. These results should be interpreted with some caution however, given the limitations of our study (e.g., small sample size and the wide age range of participants, which may have impacted on analyses that included neurodevelopmental conditions that are not typically diagnosed in early infancy e.g., ADHD). Our finding of an association between CAS and memory deficits is consistent with prior reports [46]. Speech (articulation and phonology) and language outcomes could have been impacted by hearing impairment (conductive), which occurred in $19 \%$ of the child sample. This is particularly plausible for speech outcomes as all children with impaired hearing had a speech deficit, typically articulation and/or phonological errors that occurred in the presence of CAS. A limitation of our data is that hearing status was obtained via parent-report, potentially under-representing the occurrence of hearing impairment in this cohort.

It is most probable that CAS observed in people with $16 p 11.2$ deletions is associated with specific neural and genetic markers yet to be explored in this population. While the speech phenotype associated with $16 \mathrm{p} 11.2$ deletion is largely expressed as CAS, the following profiles may be seen: (i) typical speech-sound development, (ii) isolated developmental articulation disorder or phonological delay, (iii) CAS with or without dysarthria, articulation or phonological errors, or even (iv) non-verbal presentation. Of interest would be to determine the combination of genetic and neural markers that can predict children's speech outcome. Although language impairment has been linked to the number of genomic copies of the 16p11.2 region [47] and abnormal development of neural pathways supporting language function (i.e., left arcuate fasciculus) in cases with 16 p11.2 deletion [48], the mechanisms underlying speech dysfunction for this group remain unknown.

Future research may also examine whether more severe phenotypes observed in individuals with 16p11.2 deletion (e.g., non-verbal presentations) are associated with the 'twohit' model suggested by Girirajan et al. [49] This model proposes that severe phenotypes linked to $16 \mathrm{p} 12.2$ microdeletions result from a secondary insult (e.g., an additional copy number variant, gene mutation or environmental factor). The model has been applied to children with 16p11.2 deletion [50] but it has yet to be determined how relevant it is for explaining speech outcomes.

Whilst we aimed to recruit a sample less biased than previous reports [2] and unselected for neurodevelopmental conditions (e.g., ASD), our cohort may have been biased towards children with developmental concerns due to our method of recruitment (i.e., a genetics service where individuals are often referred due to specific clinical 
indications). In addition, it is possible that being advertised as a speech and language study, our sample could be biased towards individuals with speech and language conditions. We, however, aimed to mitigate this by indicating that all individuals, even those without speech and language disorders, were eligible.

This study is the largest to date to systematically assess speech and language skills in children with 16p11.2 deletion. We confirm that CAS is a core component of the speech phenotype seen in children with $16 \mathrm{p} 11.2$ deletion. A potential extension of this work is to compare the phenotype against intra-familial controls and 16p11.2 duplication cases. Future research should be directed at examining causal pathways in order to identify the specific genetic and neural mechanisms underpinning CAS. This will provide much needed data informing the etiology of CAS given that only a small proportion of cases can currently be explained by known genetic mutations (e.g., FOXP2 and GRIN2A) [51].

Acknowledgements We thank the children and their families who participated. This study was supported by the Simons Foundation Variations in Individuals Project and the following grants from the National Health and Medical Research Council (NHMRC): Practitioner Fellowship awarded to A.M. (\#1105008) and a Centre of Research Excellence in Speech and Language NeurobioloGy (SLANG) awarded to A.M. and S.E.F. (\#1116976). S.E.F. is also supported by the Max Planck Society. E.F. and C.H. were funded by a grant from the Simons Foundation to the Simons Center for the Social Brain at MIT. This work was also supported by the Victorian Government's Operational Infrastructure Support Programme.

\section{Compliance with Ethical Standards}

Conflict of interest The authors declare that they have no conflict of interest.

\section{References}

1. Bijlsma E, Gijsbers A, Schuurs-Hoeijmakers J, et al. Extending the phenotype of recurrent rearrangements of 16p11.2: deletions in mentally retarded patients without autism and in normal individuals. Eur J Med Genet. 2009;52:77-87.

2. Fedorenko E, Morgan A, Murray E, et al. A highly penetrant form of childhood apraxia of speech due to deletion of 16p11.2. Eur J Hum Genet. 2016;24:302-6.

3. Rosenfeld J, Coppinger J, Bejjani B, et al. Speech delays and behavioral problems are the predominant features in individuals with developmental delays and 16p11.2 microdeletions and microduplications. J Neurodev Disord. 2010;2:26-38.

4. Shinawi M, Liu P, Kang SHL, et al. Recurrent reciprocal 16p11.2 rearrangements associated with global developmental delay, behavioural problems, dysmorphism, epilepsy, and abnormal head size. J Med Genet. 2010;47:332-41.

5. Hanson E, Bernier R, Porche K, et al. The cognitive and behavioral phenotype of the 16p11.2 deletion in a clinically ascertained population. Biol Psychiatry. 2015;77:785-93.

6. Laffin JJ, Raca G, Jackson CA, Strand EA, Jakielski KJ, Shriberg LD. Novel candidate genes and regions for childhood apraxia of speech identified by array comparative genomic hybridization. Genet Med. 2012;14:928-36.

7. Newbury DF, Mari F, Akha ES, et al. Dual copy number variants involving $16 \mathrm{p} 11$ and $6 \mathrm{q} 22$ in a case of childhood apraxia of speech and pervasive developmental disorder. Eur J Hum Genet, 2013;21:361-5.

8. Raca G, Baas BS, Kirmani S, et al. Childhood apraxia of speech (CAS) in two patients with 16p11.2 microdeletion syndrome. Eur J Hum Genet. 2013;21:455-9.

9. American Speech-Language-Hearing Association (ASHA). Childhood apraxia of speech [technical report]. ASHA, 2007; ww. asha.org/policy.

10. Shriberg LD, Aram DM, Kwiatkowski J. Developmental apraxia of speech: I. Descriptive and theoretical perspectives. J Speech Lang Hear Res. 1997;40:273-85.

11. Steinman KJ, Spence SJ, Ramocki MB, et al. 16p11. 2 deletion and duplication: characterizing neurologic phenotypes in a large clinically ascertained cohort. Am J Med Genet Part A. 2016;170:2943-55.

12. Bohland JW, Guenther FH. An fMRI investigation of syllable sequence production. Neuroimage. 2006;32:821-41.

13. Basilakos A, Smith K, Fillmore P, Fridriksson J, Fedorenko E. Functional characterization of the human speech articulation network. Cereb Cortex. 2017; 1-15. https://doi.org/10.1093/cercor/ bhx 100 .

14. Fernandez B, Roberts W, Chung B, et al. Phenotypic spectrum associated with de novo and inherited deletions and duplications at $16 \mathrm{p} 11.2$ in individuals ascertained for diagnosis of autism spectrum disorder. J Med Genet. 2010;47:195-203.

15. Marshall CR, Noor A, Vincent JB, et al. Structural variation of chromosomes in autism spectrum disorder. Am $\mathrm{J}$ Hum Genet. 2008;82:477-88.

16. Morgan A, Gunther T. Clinical management of articulation impairment in children. In: Dodd B, Morgan A, (eds). Intervention Case Studies of Child Speech Impairment. London: J\&R Press; 2017.

17. Dodd B. Clinical management of phonological impairment in children. In: Dodd B, Morgan A, (eds). Intervention Case Studies of Child Speech Impairment. London: J\&R Press; 2017.

18. Morgan A, Murray E. Clinical management of motor speech disorders. In: Dodd B, Morgan A, (eds). Intervention Case Studies of Child Speech Impairment.. London: J\&R Press; 2017.

19. Goldman R, Fristoe M. Goldman-Fristoe Test of Articulation-2. Minneapolis: Pearson; 2000.

20. Dodd B, Hua Z, Crosbie S, Holm A, Ozanne A. Diagnostic Evaluation of Articulation and Phonology. London: Psychological Corporation; 2002.

21. Holm A, Farrier F, Dodd B. Phonological awareness, reading accuracy and spelling ability of children with inconsistent phonological disorder. Int J Lang Commun Disord. 2008;43:300-22.

22. Morgan A, Eecen KT, Pezic A, et al. Who to refer for speech therapy at 4 years of age versus who to watch and wait. J Pediatr. 2017;185:200-4.

23. Murray E, McCabe P, Ballard KJ. A systematic review of treatment outcomes for children with childhood apraxia of speech. Am J Speech Lang Pathol. 2014;23:486-504.

24. Murray E, McCabe P, Heard R, Ballard KJ. Differential diagnosis of children with suspected childhood apraxia of speech. J Speech Lang Hear Res. 2015;58:43-60.

25. Gozzard H, Baker E, McCabe P. Requests for clarification and children's speech responses: changing "pasghetti" to "spaghetti. Child Lang Teach Ther. 2008;24:249-63.

26. Shriberg LD, Kwiatkowski J. Phonological disorders III: a procedure for assessing severity of involvement. J Speech Hear Disord. 1982;47:256-70. 
27. Hayden D, Square P. Verbal Motor Production Assessment for Children. Psychological Corporation: San Antonio; 1999.

28. McCauley RJ, Strand EA. A review of standardized tests of nonverbal oral and speech motor performance in children. Am J Speech Lang Pathol. 2008;17:81-91.

29. Robbins J, Klee T. Clinical assessment of oropharyngeal motor development in young children. $\mathbf{J}$ Speech Hear Disord. 1987;52:271-7.

30. Enderby P, Palmer R. Frenchay Dysarthria Assessment-2. Pro-Ed: Austin; 2008.

31. Wiig EH, Secord WA, Semel E. Clinical Evaluation of Language Fundamentals Preschool-2: Australian Standardised Edition. Sydney: Harcourt Assessment; 2006.

32. Semel E, Wiig EH, Secord WA. Clinical Evaluation of Language Fundamentals-4: Australian Standardised Edition. Sydney: Pearson; 2006.

33. Zimmerman IL, Steiner VG, Pond RE. Preschool Language Scales-5: Australian and New Zealand Language Adapted Edition. Sydney: Pearson; 2011.

34. Wiig EH, Semel E, Secord WA. Clinical Evaluation of Language Fundamentals-5. San Antonio: Pearson; 2013.

35. Dunn LM, Dunn DM. Peabody Picture Vocabulary Test-4. Minneapolis: Pearson; 2007.

36. Bishop D. Test for Reception of Grammar-2. London: Harcourt Assessment; 2003.

37. Wagner R, Torgesen J, Rashotte C, Pearson NA. Comprehensive Test of Phonological Processing-2. Pro-Ed: Austin; 2013.

38. Wilkinson GS, Robertson GJ. Wide Range Achievement Test-4. Lutz: Psychological Assessment Resources; 2006.

39. Wechsler D. Wechsler Abbreviated Scale of Intelligence-2. Minneapolis: Pearson; 2011.

40. Kaufman AS, Kaufman NL. Kaufman Brief Intelligence Test-2. Minneapolis: Pearson; 2004.
41. Morgan AT, Vogel AP Intervention for childhood apraxia of speech. Cochrane Database of Systematic Reviews 2008, Issue 3. Art. No.: CD006278. https://doi.org/10.1002/14651858.CD006278.pub2.

42. White SM, Morgan A, Da Costa A, et al. The phenotype of Floating-Harbor syndrome in 10 patients. Am J Med Genet Part A. 2010;152:821-9.

43. Morgan AT, Mei C, Da Costa A, et al. Speech and language in a genotyped cohort of individuals with Kabuki syndrome. Am J Med Genet Part A. 2015;167:1483-92.

44. Trouton A, Spinath FM, Plomin R. Twins early development study (TEDS): a multivariate, longitudinal genetic investigation of language and cognition, cognition and behavior problems in childhood. Twin Res. 2002;5:444-8.

45. Lewis BA, Freebairn LA, Hansen AJ, Iyengar SK, Taylor G. School-age follow-up of children with childhood apraxia of speech. Lang Speech Hear Serv Sch. 2004;35:122-40.

46. Shriberg LD, Lohmeier HL, Strand EA, Jakielski KJ. Encoding, memory, and transcoding deficits in childhood apraxia of speech. Clin Linguist Phon. 2012;26:445-82.

47. Hippolyte L, Maillard AM, Rodriguez-Herreros B, et al. The number of genomic copies at the 16p11.2 locus modulates language, verbal memory, and inhibition. Biol Psychiatry. 2016;80:129-39.

48. Berman JI, Chudnovskaya D, Blaskey L, et al. Abnormal auditory and language pathways in children with $16 \mathrm{p} 11.2$ deletion. Neuroimage Clin. 2015;9:50-7.

49. Girirajan S, Rosenfeld JA, Cooper GM, et al. A recurrent 16p12.1 microdeletion supports a two-hit model for severe developmental delay. Nat Genet. 2010;42:203-9.

50. Brisset S, Capri Y, Briand-Suleau A, et al. Inherited 1q21.1q21.2 duplication and 16p11.2 deletion: a two-hit case with more severe clinical manifestations. Eur J Hum Genet. 2015;58:497-501.

51. Deriziotis P, Fisher SE. Speech and language: translating the genome. Trends Genet. 2017;33:641. 